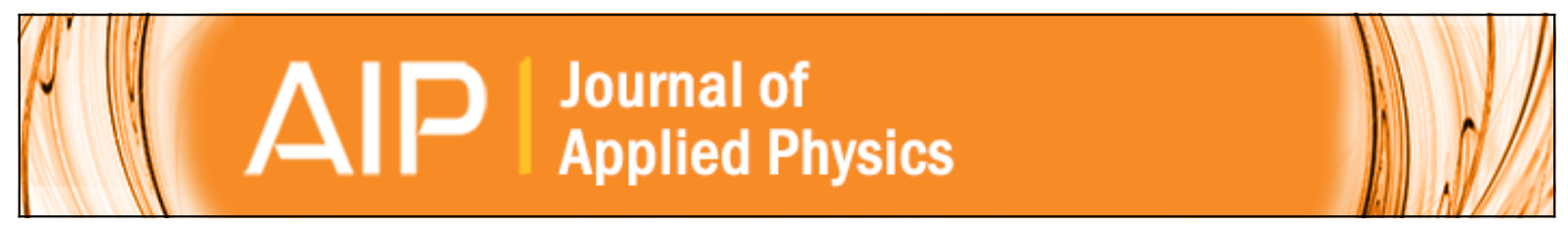

\title{
Electrical resistivity of bismuth implanted into silicon
}

A. Ferreira da Silva, Bo E. Sernelius, J. P. de Souza, and H. Boudinov

Citation: Journal of Applied Physics 79, 3453 (1996); doi: 10.1063/1.361393

View online: http://dx.doi.org/10.1063/1.361393

View Table of Contents: http://scitation.aip.org/content/aip/journal/jap/79/7?ver=pdfcov

Published by the AIP Publishing

\section{AlP Re-register for Table of Content Alerts}

\section{Create a profile. \\ Sign up today!}




\title{
Electrical resistivity of bismuth implanted into silicon
}

\author{
A. Ferreira da Silva ${ }^{\text {a) }}$ \\ Instituto Nacional de Pesquisas Espaciais, INPE, Laboratório Associado de Sensores e Materiais, LAS, \\ C.P. 515, 12201-970 São José dos Campos, SP, Brazil
}

\author{
Bo E. Sernelius \\ Department of Physics and Measurement Technology, University of Linköping, S-58183 Linköping, Sweden
}

J. P. de Souza and H. Boudinov

Instituto de Fisica, Universidade Federal do Rio Grande do Sul, 91501-970 Porto Alegre, RS, Brazil

(Received 16 May 1995; accepted for publication 13 November 1995)

\begin{abstract}
We investigate the electrical properties of Bi-doped Si samples, prepared by ion implantation, in a range of concentrations around and above the metal-nonmetal transition. Comparison between experimental and theoretical values of the resistivity brought out that in these samples a similar behavior is observed as for other $n$-doped $\mathrm{Si}$, thus confirming the results obtained in the same range of impurity concentration, i.e., $\rho(\mathrm{Sb})<\rho(\mathrm{P})<\rho(\mathrm{As})<\rho(\mathrm{Bi})$. (C) 1996 American Institute of Physics. [S0021-8979(96)05404-X]
\end{abstract}

\section{INTRODUCTION}

The theoretical and experimental investigations of the transport properties of heavily doped semiconductors continue to be a topic of considerable interest. ${ }^{1-13}$ Recently the systems $\mathrm{Si}: \mathrm{P},{ }^{2-5,10-13} \mathrm{Si}: \mathrm{As},{ }^{3,12,13} \mathrm{Si}: \mathrm{Sb},{ }^{3} \mathrm{Si}: \mathrm{Bi},{ }^{3} \mathrm{Si}: \mathrm{B},{ }^{1,9}$ and Si doubly doped with $\mathrm{P}$ and As donors (Si:P, As), ${ }^{6}$ and $\mathrm{P}$ donors and $\mathrm{B}$ acceptors $(\mathrm{Si}: \mathrm{P}, \mathrm{B}),{ }^{6-8}$ and other pair systems $^{6,14,15}$ have again demonstrated the interest of such investigations. Nevertheless, some lack of experimental and theoretical information exists, particularly for $\mathrm{Bi}$, the last dopant of the $\mathrm{V}$ column of the periodic table, which has been observed to have a low equilibrium solid solubility limit in silicon crystal. ${ }^{3,16}$ It is worthwhile to point out that the $\mathrm{Si}: \mathrm{Bi}$ system has not been given much attention probably because of its large ionization energy and smaller effective Bohr radius, i.e., $E_{I}=71 \mathrm{meV}$ and $a^{*}=8.9 \AA$, respectively, compared to the three donors, P, As, and Sb. With such values of $E_{I}$ and $a^{*}$ the theory of the effective mass was expected to be less applicable to the $\mathrm{Si}: \mathrm{Bi}$ system. ${ }^{3}$ Owing to the smaller value of $a^{*}$ one expects that a higher donor concentration will be required to reach a metallic regime, i.e., above the metal-nonmetal (MNM) transition.

The aim of this work is to investigate the Hall mobility $\mu_{H}$ and resistivity $\rho$ of $\mathrm{Si}: \mathrm{Bi}$ in a range of doping around and above the MNM transition. The experimental procedure is at room temperature with the samples prepared by ion implantation, which is an important method of making semiconductor devices. ${ }^{16}$

The theoretical calculations are carried out at a similar doping regime. The impurity critical concentration $N_{c}$ for the MNM transition has been proposed to describe the specific feature of the transition. The values of $N_{c}$ were found to vary from 1.0 to $1.8 \times 10^{19} \mathrm{~cm}^{-3}$.

In the measured resistivity of the $\mathrm{Si} B \mathrm{Bi}$ system we observe a continuous increase of resistivity around $N_{c}$, going below the metallic region. This behavior is more accentuated in $\mathrm{Si}: \mathrm{Bi}$ compared to other $n$-type-doped $\mathrm{Si}$

a)Electronic mail: ferreira@las.inpe.br systems. ${ }^{3,10-13,17,18}$ In Si:P, for instance, increasing temperature, from 4.2 to $300 \mathrm{~K}$, results in a lower resistivity below the MNM transition. ${ }^{19,20}$ There is a crossover of $\rho$ around $N_{c}$ for this range of temperatures. Above this value $\rho$, at $4.2 \mathrm{~K}$, presents a similar behavior for those higher temperatures, i.e., 77 and $300 \mathrm{~K}$, respectively. ${ }^{12,19,20}$ The results obtained confirm the general features of the resistivity, i.e., $\rho(\mathrm{Sb})<\rho(\mathrm{P})<\rho(\mathrm{As})<\rho(\mathrm{Bi}) ., 21,22$

\section{EXPERIMENTAL DETAILS}

The Si wafers used in this work were of $p$ type and (100) oriented with resistivity in the range of 16-25 $\Omega \mathrm{cm}$ Van der Pauw structures ${ }^{23}$ having low sheet resistance areas $(<20$ $\Omega / \square$, phosphorus doped) were implanted at nominal room temperature with $\mathrm{Bi} \pm$. Five implantations with energies of $35,70,120,200$, and $360 \mathrm{keV}$ were accumulated in each sample with proper doses to result in a plateaulike depth profile of Bi with $\sim 5 \%$ deviation up to the depth of $0.15 \mu \mathrm{m}$, according to TRIM simulation. ${ }^{24}$

Samples with $\mathrm{Bi}$ concentrations of $5 \times 10^{17}, 5 \times 10^{18}$, $1 \times 10^{19}, 5 \times 10^{19}, 1 \times 10^{20}$, and $5 \times 10^{20} \mathrm{~cm}^{-3}$ were prepared and labeled as samples 1, 2, 3, 4, 5, and 6, respectively. For sample 6 the implanted $\mathrm{Bi}$ doses are $5 \times 10^{14} \mathrm{~cm}^{-2}$ (at 35 $\mathrm{keV}$ ), $7 \times 10^{14} \mathrm{~cm}^{-2}$ (at $\left.70 \mathrm{keV}\right), 8 \times 10^{14} \mathrm{~cm}^{-2}$ (at $120 \mathrm{keV}$ ), $1.4 \times 10^{15} \mathrm{~cm}^{-2}$ (at $200 \mathrm{keV}$ ), and $2.5 \times 10^{15} \mathrm{~cm}^{-2}$ (at 360 $\mathrm{keV})$. For the samples 1, 2, 3, 4, and 5 these doses were reduced by a factor of 1000, 100, 50, 10, and 5 times, respectively.

The damage annealing and the electrical activation of $\mathrm{Bi}$ was performed at $600{ }^{\circ} \mathrm{C}$ for $30 \mathrm{~min}$ in an inert atmosphere. Since $\mathrm{Bi}+$ doses for samples 1,2 , and 3 were below the threshold for amorphization a second annealing step at $900{ }^{\circ} \mathrm{C}$ for $60 \mathrm{~s}$ was performed to enhance the electrical activation of $\mathrm{Bi}$.

The van der Pauw devices were electrically characterized by sheet resistivity $R(\Omega / \square)$ and Hall measurements at room temperature. 
TABLE I. Room-temperature measurements of Si:Bi.

\begin{tabular}{lccccc}
\hline \hline Sample & $R(\Omega / \hbar)$ & $\mu\left(\mathrm{cm}^{2} / \mathrm{V} \mathrm{s}\right)$ & $n_{s}\left(\mathrm{~cm}^{-2}\right)$ & $N_{d}\left(\mathrm{~cm}^{-3}\right)$ & $\rho(\Omega \mathrm{cm})$ \\
\hline 1 & 3424 & 535 & $3.4 \times 10^{12}$ & $3.0 \times 10^{17}$ & 0.039 \\
2 & 1112 & 277 & $2.0 \times 10^{13}$ & $1.8 \times 10^{18}$ & 0.012 \\
3 & 708 & 189 & $4.7 \times 10^{13}$ & $4.0 \times 10^{18}$ & 0.0083 \\
4 & 302 & 77 & $2.7 \times 10^{14}$ & $2.2 \times 10^{19}$ & 0.0037 \\
5 & 187 & 59 & $5.7 \times 10^{14}$ & $4.8 \times 10^{19}$ & 0.0022 \\
6 & 99 & 38 & $1.7 \times 10^{15}$ & $1.4 \times 10^{20}$ & 0.0012 \\
\hline \hline
\end{tabular}

\section{RESULTS AND DISCUSSION}

The room-temperature impurity resistivity is calculated from the generalized Drude approach (GDA), ${ }^{25,26}$

$$
\rho(w)=\frac{-i m^{*} w}{N_{d} e^{2}}+i \frac{2}{3 \pi N_{d} k w} \int_{0}^{\infty} d q q^{2}\left(\frac{1}{\epsilon(q, w)}-\frac{1}{\epsilon(q, 0)}\right) .
$$

This expression is assumed to be valid for all frequencies $w$, especially for zero frequency which is the limit of interest here. In Eq. (1) $\epsilon(q, 0)$ is the static temperaturedependent random-phase-approximation (RPA) dielectric function of the donor electrons, $m^{*}$ is the effective mass, and $k$ is the dielectric constant of the material. ${ }^{25,26}$

For the sake of comparison the low-temperature impurity resistivity $\rho=1 / \sigma$ (inverse of the conductivity $\sigma$ ) is also calculated from the Sommerfeld expansion, where we keep only the lower-order terms. Therefore, we can use such an expansion for $T<10 \mathrm{~K}$, which is the validity of this calculation, to obtain ${ }^{3,5}$

$$
\sigma=\sigma_{0}+\Delta \sigma
$$

where $\sigma_{0}$ is the zero temperature conductivity derived from the Kubo formalism, given by ${ }^{3,5}$

$$
\sigma_{0}=\frac{e N_{v}}{3 \hbar} \int \sum\left(E_{F}, N_{d}\right)\left(\frac{d f(E)}{d E}\right) d E
$$

and

$$
\Delta \sigma=f\left[T, D\left(E_{F}\right)\right] .
$$

In the above equations, $N_{V}$ is the number of valleys of the conduction band $\left(N_{V}=6\right.$ for $\left.\mathrm{Si}\right),{ }^{3} N_{d}$ is the donor impurity concentration, $E_{F}$ is the Fermi energy, $D\left(E_{F}\right)$ is the density of states at $E_{F}$, and $f(E)$ is the Fermi distribution function.

For the MNM transition four criteria have been used to determine $N_{c}$, based mainly on use of a regular lattice, a randomlike distribution of impurities, a disordered one-band model, and the Hubbard model. The values of $N_{c}$ vary from 1.0 to $1.8 \times 10^{19} \mathrm{~cm}^{-3} .3$ The latter value of $N_{c}$, corresponding to the two first criteria, is closer to the value obtained from the general expression of the Mott criterion (i.e., $N_{c}^{1 / 3} a^{*} \cong 0.25$ ) for the MNM transition. ${ }^{27}$ We adopt this value.

In Table I we show the measured values of the samples with concentration varying from $3.0 \times 10^{17}$ to $1.4 \times 10^{20}$ $\mathrm{cm}^{-3}$. The resistivity is calculated by $\rho=1 / q \mu N$ and the Hall coefficient by the relation $R_{H}=1 / N q$, where $q$ is the electrical charge. It means that the Hall scattering factor $A=1$,

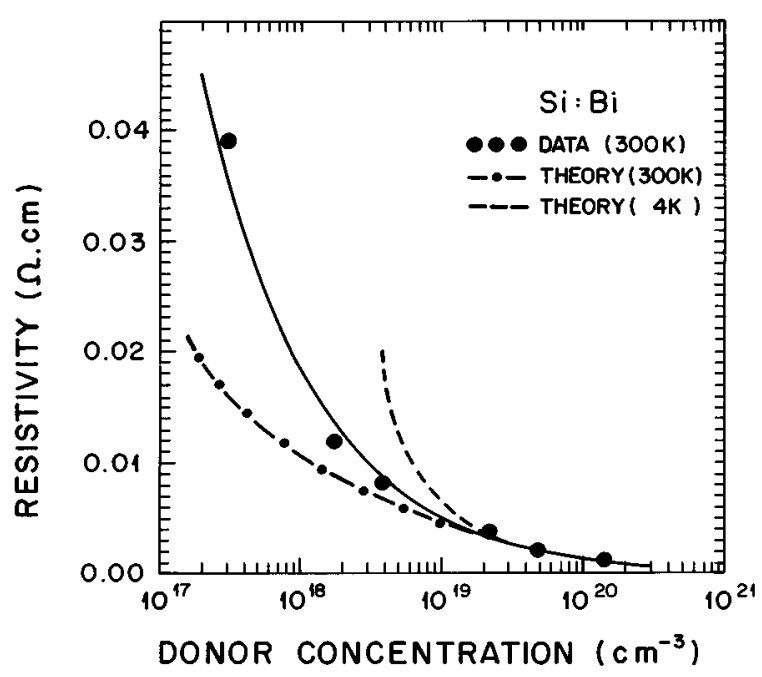

FIG. 1. The room-temperature resistivity $\rho$ of $\mathrm{Si}: \mathrm{Bi}$ as a function of donor impurity concentration $N_{d}$. The dotted-dashed and dashed lines represent our calculations at 300 and $4 \mathrm{~K}$, using Eqs. (1) and (2), respectively.

which is a good approximation for heavily doped semiconductors. ${ }^{13}$ The carrier concentration $N$ is identified with $N_{d}$.

In Fig. 1 we plot the resistivity of $\mathrm{Si}: \mathrm{Bi}$ as a function of impurity concentration for both experimental and theoretical results. The experimental data, at room temperature, are represented by solid circles. The line joining the circles is obtained by fitting of the equation

$$
\rho=b N^{-a},
$$

where $a=0.5517$ and $b=1.582 \times 10^{-8}$. We observe a continuous increase of the resistivity with decreasing doping regime around $2 \times 10^{19} \mathrm{~cm}^{-3}$, where, at very low temperature, one expects a MNM transition. ${ }^{3}$

This continuous increase of resistivity at room temperature is more accentuated than, for instance, in the $\mathrm{Si}: \mathrm{P}$

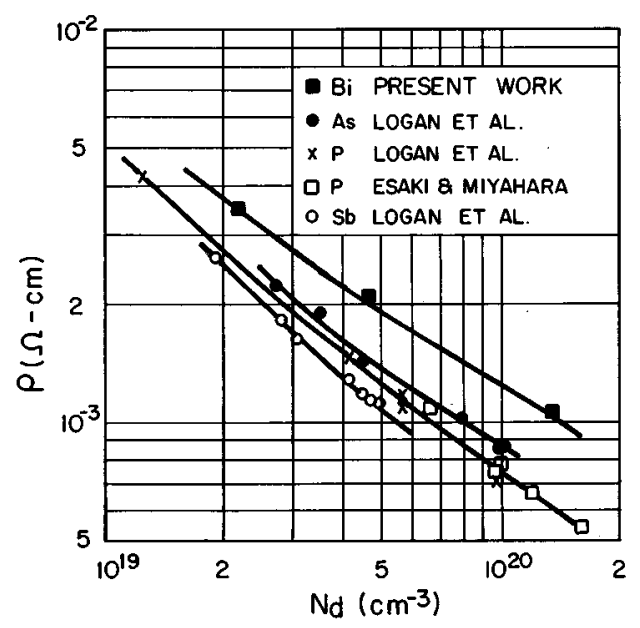

FIG. 2. Log-log plot of resistivity vs impurity concentration at room temperature, for silicon samples doped, respectively, with bismuth, arsenic, phosphorus, and antimony. The data of $(-\mathrm{As},(\times) \mathrm{P}$, and $(\bigcirc) \mathrm{Sb}$ are from Logan and co-workers (Ref. 21) and ( $\square$ ) P are from Ref. 22. 


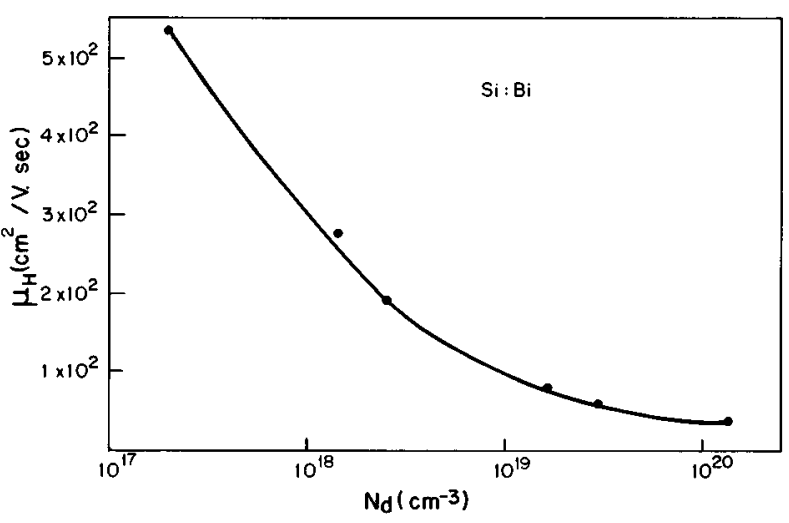

FIG. 3. Hall mobility $\mu_{H}$ vs donor impurity concentration of $\mathrm{Si}: \mathrm{Bi}$ at room temperature.

system. ${ }^{19,20}$ We may argue that this is due to its low ionization energy of $71 \mathrm{meV}$, compared to $45 \mathrm{meV}$ of Si:P, which will lead to higher values of $N_{c}$ at the MNM transition.

In Fig. 1 we also observe a similar behavior above the MNM transition for both calculations, i.e., $300 \mathrm{~K}$ and $4 \mathrm{~K}$, using Eqs. (1) and (2), respectively, as one expects for the metallic regime. For the temperature of $300 \mathrm{~K}$ the agreement between theory and experiment, above $10^{18} \mathrm{~cm}^{-3}$, looks quite good.

The doping of $\mathrm{Si}$ with $\mathrm{Sb}, \mathrm{P}, \mathrm{As}$, or Bi gives rise at low concentrations to a set of discrete donor levels with bound states whose ionization energies (which directly determine their effective Bohr radia) are about 43, 45, 53, and $71 \mathrm{meV}$, respectively. ${ }^{3,28}$ Their respective effective Bohr radia are about $16,15,13$, and $9 \AA .{ }^{3,28}$ As the concentration of donors is increased, the overlap and hopping between nearby states and the random arrangements of adjacents centers cause the bound-state energies to be spread into a band which is eventually separated from the host conduction band. Considering the effective Bohr radius to vary from the lower to the larger value, i.e., $a^{*}(\mathrm{Bi})$ to $a^{*}(\mathrm{Sb}), \sigma$ moves toward larger values in the following order: $\sigma(\mathrm{Sb})>\sigma(\mathrm{P})>\sigma(\mathrm{As})>\sigma(\mathrm{Bi})$. This is as expected because a large $a^{*}$ (i.e., small ionization energy) causes a larger overlap and, consequently, a bigger conductivity. As a consequence $N_{c}$ moves toward lower values, i.e., $\quad N_{c}(\mathrm{Sb})<N_{c}(\mathrm{P})<N_{c}(\mathrm{As})<N_{c}(\mathrm{Bi}), \quad$ where $\quad N_{c}(\mathrm{Sb})$ $=3.0 \times 10^{18} \quad \mathrm{~cm}^{-3}, \quad N_{c}(\mathrm{P})=3.7 \times 10^{18} \quad \mathrm{~cm}^{-3}, \quad N_{c}(\mathrm{As})$ $=(6.4-8.5) \times 10^{18} \mathrm{~cm}^{-3}$, and $N_{c}(\mathrm{Bi})=1.8 \times 10^{19} \mathrm{~cm}^{-3} .3,28$

In Fig. 2 we compare the resistivity versus the impurity concentration, at $300 \mathrm{~K}$, for the $\mathrm{Si}: \mathrm{Bi}$ system with those of the other three systems, i.e., Si:As, Si:P, and $\mathrm{Si}: \mathrm{Sb}$. Above
$10^{19} \mathrm{~cm}^{-3}$ we confirm the general feature observed for resistivity, i.e, $\rho(\mathrm{Bi})>\rho(\mathrm{As})>\rho(\mathrm{P})>\rho(\mathrm{Sb}){ }^{3,21}$

Figure 3 shows the room-temperature Hall mobility as a function of bismuth concentration. We observe that the qualitative and quantitative values, above $10^{17} \mathrm{~cm}^{-3}$, are similar to those obtained by Mousty, Ostoja, and Passan for Si:P. ${ }^{29}$ Above $N_{d}=2 \times 10^{19} \mathrm{~cm}^{-3}$ the values of $\mu_{H}=\mu q \approx 10^{2}$ $\mathrm{cm}^{2} / \mathrm{V} \mathrm{s}$ are close to the theoretical prediction. ${ }^{3}$

In the absence of a complete study in $\mathrm{Si}: \mathrm{Bi}$ comparable, at least, to the Mousty and co-workers work ${ }^{29}$ in $\mathrm{Si}: \mathrm{P}$, we conclude that further analysis of this system is certainly very interesting. Such work is under consideration even at very low temperature and will be reported in the near future.

${ }^{1}$ S. Bogdanovich, Peihua Dai, M. P. Sarachik, and V. Dobrosavljevic, Phys. Rev. Lett. (to be published); S. Bogdanovich, Peihua Dai, M. P. Sarachik, V. Dobrosavljevic, and G. Kotliar (unpublished).

${ }^{2}$ Peihau Dai, Youzhu Zhang, and M. P. Sarachik, Phys. Rev. B 49, 14039 (1994).

${ }^{3}$ A. Ferreira da Silva, J. Appl. Phys. 76, 5249 (1994).

${ }^{4}$ M. Hornung, A. Ruzzu, H. G. Schlager, H. Stupp, and H. V. Löhneysen, Europhys. Lett. 28, 43 (1994).

${ }^{5}$ A. Ferreira da Silva, Phys. Rev. B 48, 1921 (1993).

${ }^{6}$ A. Ferreira da Silva, Phys. Rev. B 50, 11216 (1994).

${ }^{7}$ U. Thomanschefsky and D. F. Holcomb, Phys. Rev. B 45, 13356 (1992).

${ }^{8}$ M. J. Hirsch, D. F. Holcomb, R. N. Bhatt, and M. Paalanen, Phys. Rev. Lett. 68, 1418 (1992).

${ }^{9}$ Peihua Dai, Youzhu Zhang, and M. P. Sarachik, Phys. Rev. Lett. 66, 1914 (1991); 67, 136 (1991).

${ }^{10}$ U. H. Thomanschefsky, Ph.D. thesis, Cornell University, 1990.

${ }^{11}$ U. H. Thomanschefsky (unpublished).

${ }^{12}$ M. J. Hirsch and D. F. Holcomb, Phys. Rev. B 33, 2520 (1986).

${ }^{13}$ P. F. Newman, M. J. Hirsch, and D. F. Holcomb, J. Appl. Phys. 58, 3779 (1985).

${ }^{14}$ G. -J. Yi and G. F. Neumark, Phys. Rev. B 48, 17043 (1993).

${ }^{15}$ G. -J. Yi, J. A. Tuchman, and G. F. Neumark, Appl. Phys. Lett. 58, 520 (1991).

${ }^{16}$ J. P. de Souza and P. F. P. Fichtner, J. Appl. Phys. 74, 119 (1993), and references therein.

${ }^{17}$ J. del Alamo and R. M. Swanson, J. Appl. Phys. 57, 2314 (1985).

${ }^{18}$ W. R. Thurber, R. L. Mattis, Y. M. Liu, and J. J. Filliben, J. Electrochem. Soc. 127, 1807 (1980).

${ }^{19}$ C. Yamanouchi, K. Mizuguchi, and W. Sasaki, J. Phys. Soc. Jpn. 22, 859 (1967).

${ }^{20}$ W. Sasaki, J. Phys. Soc. Jpn. 49, Suppl. A, 31 (1980).

${ }^{21}$ R. A. Logan, J. F. Gilbert, and F. A. Trumbore, J. Appl. Phys. 32, 131 (1961).

${ }^{22}$ L. Esaki and Y. Miyahara, Solid-State Electron. 1, 12 (1960).

${ }^{23}$ L. J. Van der Pauw, Philips Res. Rep. 13, 1 (1958).

${ }^{24}$ J. F. Ziegler, J. P. Biersack, and U. Littmark, The Stopping and Ranges of Ion in Solids (Pergamon, New York, 1985), Vol. 1.

${ }^{25}$ B. E. Sernelius, Phys. Rev. B 40, 12438 (1989).

${ }^{26}$ B. E. Sernelius and E. Söderström, J. Phys. C 3, 1493 (1991).

${ }^{27}$ P. P. Edwards and M. Sienko, Phys. Rev. B 17, 2575 (1978).

${ }^{28}$ A. Ferreira da Silva, Phys. Scr. T 14, 27 (1986).

${ }^{29}$ F. Mousty, P. Ostoja, and L. Passan, J. Appl. Phys. 45, 4576 (1974). 